\title{
Analyse pollinique des miels de l'Ontario, Canada
}

\author{
M.J. Feller-Demalsy et J. Parent \\ Université du Québec à Rimouski, 300, allée des Ursulines, Rimouski, QC, Canada, G5L 3A1
}

(reçu le 3-7-1987, accepté le 22-10-1988)

Résumé - L'étude a été menée sur 17 miels récoltés pour la plupart en 1983 et provenant du sud de l'Ontario (Canada) où est concentrée l'activité apicole. Les résultats révèlent six taxons caractéristiques des miels de cette région : Lotus corniculatus (lotier corniculé), Trifolium hybridum / T. repens (trèfle hybride / trèfle blanc), Melilotus (mélilot), Salix (saule), Trifolium pratense (trèfle rouge) et le type Solidago (verge d'or et aster) présents dans plus de $80 \%$ des échantillons. La moitié des miels sont unifloraux avec deux types dominants : lotier et trèfle. Un miel de tilleul (Tilia) a été relevé mais sa production paraît sporadique. Les indicateurs de miellat sont réduits. Des caractéristiques locales ont été décelées.

miel — analyse pollinique - Canada

Summary - Pollen analysis of honeys from Ontario, Canada. This study was carried out on 17 samples of honey from the south of the province of Ontario, Canada, where apiculture is concentrated. Most of the samples were collected in 1983 (Fig. 1).

Most of the honeys sampled had a moderate number of pollen grains $110 \mathrm{~g}$ of honey. Pollen analysis showed 60 pollen forms, of which 50 came from melliferous and 10 from non-melliferous plants (Tables I, II and III). The number of pollen types in honeys encompassed a large range of classes from 6-10 to 31-35 forms (Fig. 2). The average number of pollen types per honey was 21, higher than in honeys from the Prairie provinces and from Quebec, Canada, probably because of the southern situation of this part of Ontario.

Six pollen forms present in $80 \%$ and more of the samples are characteristic of this region : Lotus, Trifolium hybridum / T. repens, Melilotus, Salix, Trifolium pratense and the Solidago type. Half of the honey samples were unifloral ( $>45 \%$ pollen of one taxon in sample, cf. Louveaux et al., 1978). The predominance of Lotus and of Trifolium hybridum / T. repens were the most numerous (Table IV). The unique predominance of Brassicaceae (others) seems to be fortuitious. One case of honey from Tilia, taxon, of which the pollen is normally under-represented, was shown. This was confirmed by the number of grains / $10 \mathrm{~g}$ of honey (Table $\mathrm{V}$ ).

Among the very frequent pollen types ( $50 \%$ ), besides Lotus and Trifolium hybridum / T. repens, Melilotus and the Solidago type fall in the class of secondary pollen (16-45\%) (Table IV); Salix, Trifolium pratense, the fruit trees, Rubus, Rhus typhina and the Rosaceae (folded) enter into the class of important minor pollens (3-15\%).

Honeys of Trifolium hybridum / T. repens were collected throughout southern Ontario while the honeys of Lotus seemed to be characteristic of the westem part of this region. The pollen of Echium vulgare seemed to be characteristic of the eastern region; the pollens of Viburnum, Lythrum salicaria and Cornus were very frequent ( $>50 \%$ ) in honeys from the territory adjacent to Lake Ontario and to St-Lawrence River to the Ottawa River (Table VI). Pollens of non-melliferous plants were more frequent in honeys from Ontario than in honeys from the Prairie provinces. 
In floral composition, the honeys from Ontario were close to honeys from the west of Quebec and of some honeys from the eastem Manitoba, Canada. Lotus corniculatus pollen was previously unreported as a characteristic component of honey.

honey - pollen analysis - Canada

Zusammenfassung - Pollenanalyse von Honigen aus Ontario, Kanada. Die Analyse wurde an 17 Proben aus dem Süden der Provinz Ontario, wo die Bienenhaltung konzentriert ist, durchgeführt. Die meisten Proben wurden im Jahr 1983 gesammelt (Abb. 1).

Die Mehrzahl der Honigproben hatte eine mittelmäBige Anzahl an Pollenkörnem pro $10 \mathrm{~g}$ Honig. Die Pollenanalyse ergab 60 verschiedene Pollenformen, von denen 50 von honigliefernden Pflanzen stammen und 10 von Ptlanzen, die keinen Honig liefern (Tab. I, II und III). Die Anzahl an Pollentypen in den Honigen varierte über eine große Anzahl von Klassen (6-10 bis 30-35 Formen/Honig) (Abb. 2). Die durchschnittliche Anzahl von Pollentypen/Honig lag bei 21, also hober als in den Honigen der Prärie-Provinzen und von Quebec, wahrscheinlich wegen der südlichen Lage dieses Teils von Ontario.

Sechs Pollenformen, die in $80 \%$ und mehr der Proben vorhanden waren, sind charakteristisch för die Region : Lotus, Trifolium hybridum / T. repens, Melilotus, Salix, Trifolium pratense und der Solidago Typ. Etwa die Hälfte der Proben war unifloral (> 45\% Pollen eines Taxons in der Probe). Die Vorherrschaft von Lotus und Trifolium hybridum / T. repens war am hăufigsten (Tab. IV). Die einmalige Vorherrschaft von Brassicaceen (andere) scheint rein zufallig zu sein. Ein Fall von Lindenhonig (Tilia), bei dem normalerweise der Pollen unterrepäsentiert ist, konnte nachgewiesen werden. Die Anzahl der Pollenkömem pro $10 \mathrm{~g}$ Honig bestätigte diese Unterrepräsentierung (Tab. V).

Neben den sehr häufigen Pollentypen (> 50\%) wie Lotus und Trifolium hybridum / T. repens, fa/Ien Melilotus und der Solidago-Typ in die Klasse des Begleitpollens (16-45\%) (Tab. M); Salix, Trifolium pratense, die Obstbäume, Rubus, Rhus typhina und die Rosaceen (gefaltet) sind in der Klasse von wichtigen Einzelpollen (1-15\%) zu finden.

Honige von Trifolium hybridum / T. repens wurden im ganzen Süden von Ontario, Kanada, gefunden, die Lotus-Honige scheinen charakteristisch für den westlichen Teil der Region zu sein. Pollen von Echium vulgare sind für die Ostregion charakteristisch, Pollen von Viburnum, Lythrum salicaria und Cornus wurden häufig (> 50\%) in Honigen aus dem Gebiet zwischen Ontario-See und den Flüssen Sankt Lorenz und Ottawa gefunden (Tab. VI).

Pollen von Pflanzen, die keinen Honig liefern, wurden häufiger in Honigen aus Ontario als in Honigen der Prärie-Provinzen gefunden.

Die forale Komposition der Honige aus Ontario, Kanada, war ähnlich der von Honigen aus dem Westen von Quebec und aus Ost-Manitoba, Kanada. Als Hauptcharakteristikum ist der Pollen von Lotus corniculatus anzusehen, worauf bis jetzt noch nicht hingewiesen wurde.

Honlg - Pollenanalyse - Kanada

\section{Introduction}

Les miels de l'Ontario ont fait l'objet de peu d'études quant à l'analyse microscopique des sédiments. Louveaux (1966) a effectué l'analyse pollinique de 6 miels de cette province dans une étude de 40 échantillons canadiens. Adams et al. (1979 et 1981) fournissent l'analyse polli- nique de 7 miels de la région de Guelph dans le cadre d'une étude des nectars récoltés par les abeilles. Austin (1958) a sélectionné, en vue d'analyse chimique, 3 miels caractéristiques de l'Ontario dont l'identification des sources florales a été effectuée par analyse pollinique ou par l'observation des sources florales sur le terrain. Des renseignements sur les plantes mellifères de cette province sont 
fournis par Townsend et Burke (1952), Jamieson (1958), Pellet (1977) et Ramsay (1987).

L'activité apicole de l'Ontario est concentrée dans le sud de la province. Partiellement encadrée par les Grands Lacs, cette région s'étend entre les $42^{\circ}$ et $46^{e}$ degrés de latitude Nord. Possédant les terres les plus fertiles de l'Ontario, elle en est aussi la principale région agricole avec les neuf dixièmes de l'agriculture (Benton, 1968). Sa moitié Ouest, la plus propice à cette activité, permet des cultures spécialisées diverses; dans sa moitié Est, moins favorable, l'exploitation agricole est surtout consacrée à l'élevage du bétail (Simpson-Lewis et al., 1980). Le rendement par ruche en Ontario se situe près de la moyenne de production du Canada (Statistique Canada, 1984) comme l'avait déjà évalué Crane (1966).

Cette étude vise à définir la composition pollinique des miels de l'Ontario. Cette publication est la sixième d'une série se rapportant à l'analyse pollinique des miels du Canada.

\section{Matériel et Méthodes}

\section{L'échantillonnage}

L'étude porte sur 17 miels dont 10 nous sont parvenus par l'intermédiaire de la direction de la section apicole du ministère de l'Agriculture de l'Ontario mais envoyés directement par les apiculteurs qui ont recu en retour l'analyse pollinique de leur produit. Un échantillon nous a été fourni par l'intermédiaire du «Laboratoire d'expertise et d'analyse alimentaire du ministère de l'Agriculture des Pêcheries et de l'Alimentation du Québec." Six miels ont été achetés sur place. Ces échantillons proviennent du sud de la province. La majorité des miels ont été récoltés en 1983 mais quelques-uns proviennent des années 1979 ( $n^{\circ} 264$ et 492), 1980 $\left(n^{\circ} 13,14\right.$ et 15$)$ et $1982\left(n^{\circ} 328\right)$. La Figure 1 donne la liste des lieux de production des échantillons et leur localisation géographique.
Les sites de production ont été localisés avec précision grâce au Répertoire géographique du Canada (Comité permanent canadien des noms géographiques, 1974). L'échantillonnage a été effectué pendant l'extraction du miel qui, au Canada, est faite par centrifugation.

\section{Préparation du matériel}

Les méthodes de préparation du matériel, les méthodes d'analyse et le traitement des résultats ont été décrits dans la première d'une série de publications sur les miels canadiens (FellerDemalsy, 1979). Des précisions et quelques modifications ont été apportées ultérieurement (Feller-Demalsy et al., 1987, a) : les classes d'abondance des pollens dans les miels (CAP10), c'est-à-dire le nombre de grains contenus dans $10 \mathrm{~g}$ de miel, ont été déterminées avant le montage du sédiment centrifugé dans la glycérine-gélatine au moyen d'un hémacytomètre (Neubauer Ultra Plan Lite S/P); l'identification des pollens a été effectuée au grossissement de $1250 \mathrm{X}$ sur 300 grains de plantes mellifères; les pollens de plantes non mellifères ainsi que les indicateurs de miellat ont été comptés séparément.

La fréquence de distribution des pollens a été déterminée suivant le nombre de miels dans lesquels les différents taxons sont relevés : "très fréquent" ( $>50 \%$ ), «fréquent" $(20-50 \%)$, wpeu fréquent" $(10-20 \%)$ et urare" $(<10 \%)$. Les classes de fréquence des pollens dans les miels ont été distinguées suivant les recommandations de Louveaux et al. (1978). La présence d'un "pollen dominant" dans un miel permet, dans la plupart des cas, de le considérer comme miel «unifloral». S'il n'y a pas de pollen dominant, le miel est considéré comme "toutes fleurs".

\section{Identification des taxons}

L'identification des pollens a été effectuée au plus proche taxon possible. Le terme atype" est utilisé pour indiquer tous les genres ou espèces représentés par le même type morphologique. Les types utilisés sont ceux de Moore et Webb (1978) avec une exception : la dénomination type Anthemis est remplacée par Chrysanthemum leucanthemum qui est du méme type mais est plus fréquent dans les régions étudiées. L'expression cf. (confer) est utilisée lorsqu'il y a une grande similarité morphologique sans identification certaine (Birks, 1973). Le terme autres est utilisé pour désigner les espèces non déterminables de certains 


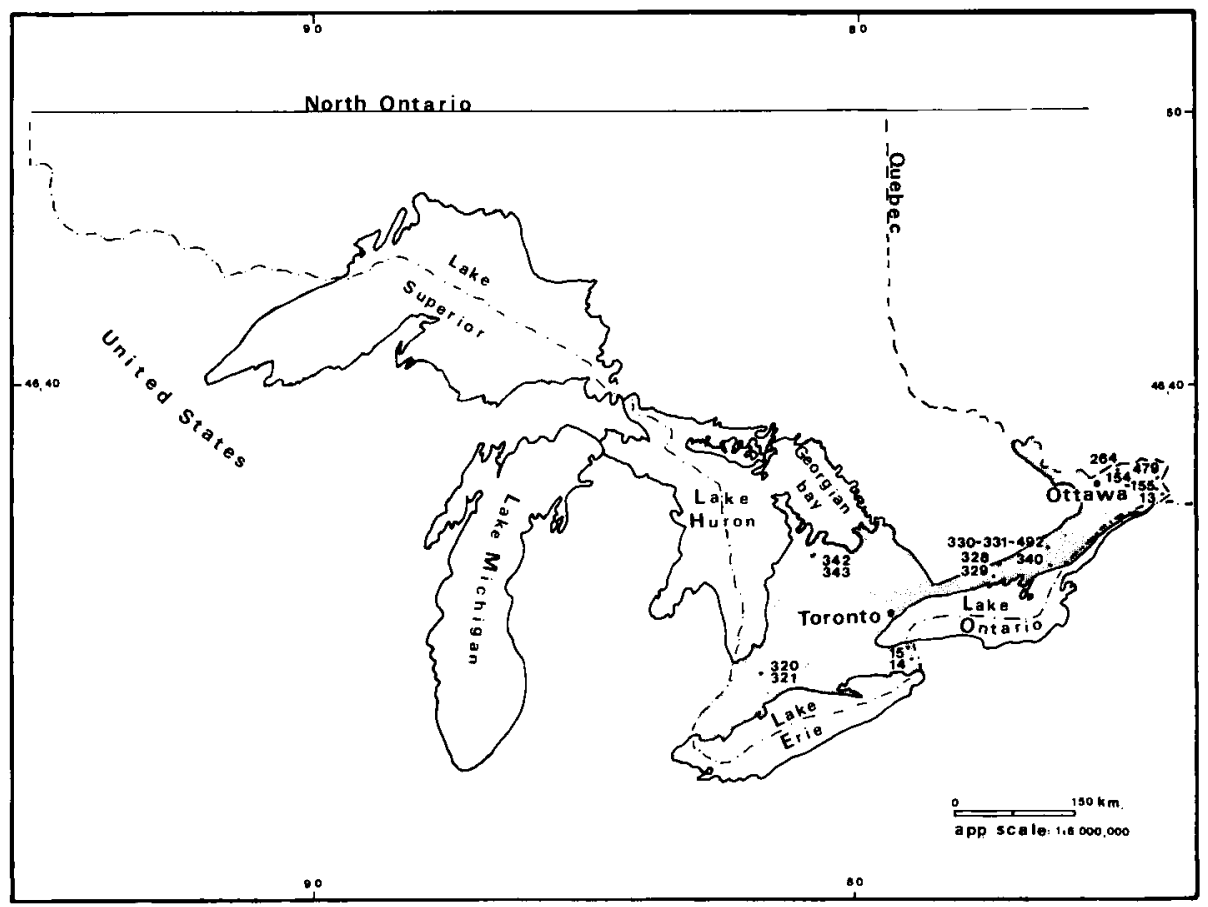

Fig. 1. Sites de production des miels ontariens soumis à l'analyse pollinique. Les frontières internationales sont indiquées par des traits alternant avec des points, les limites provinciales par des traits interrompus et le territoire agricole est rempli par un fin pointillé. Le territoire est divisé pour l'étude en trois régions : l'ouest, le centre et l'est. A l'ouest, les sites sont : 14 - Niagara Falls; 15 - Agincourt; 320 - Alviston; 321 - Alviston; 342 - Bruce County; 343 - Grey County. Au centre, les sites sont : 328 - Trenton; 329 - Trenton; 330 - Seeley's Bay; 331 - Seeley's Bay; 340 - Town of King; 492 - Seeley's Bay. A l'est, les sites sont : 13 - Long Sault; 154 - Green Valley; 155 - Green Valley; 264 Navan; 479 - Alexandria.

groupes systématiques : les espèces de Brassicaceae autres que Brassica kaber; des espèces de Vicia autres que Vicia cracca et Vicia faba; des espèces de Acer autres que Acer spicatum, Acer rubrum, Acer saccharum, Acer pensylvanicum et Acer saccharinum; des espèces de Rosaceae autres que Rubus, les arbres fruitiers, Spiraea, Fragaria, Potentilla, Sanguisorba canadensis.

Certains pollens de Rosaceae n'ont pu être identifiés avec certitude par suite des replis de l'exine. II s'agit probablement d'arbres fruitiers ou de Rubus. L'identification des pollens a principalement été effectuée d'après la colluction des pollens de référence du laboratoire de mélissopalynologie de l'Université du Québec à Rimouski. Cette collection a été enrichie pour cette étude à partir de spécimens d'herbier et de plantes récoltées sur le terrain.

\section{Résultats}

Les nombres de grains par $10 \mathrm{~g}$ de miel (CAP-10) varient de pauvres $(<20000)$ à riches (100 000 a 500000 grains). Toutefois, le plus grand nombre de miels $(64,7 \%)$ ont un CAP-10 moyen (20 000 100000 grains); $17,7 \%$ des miels sont pauvres en grains et un pourcentage identique est riche en grains. 
Le Tableau I donne le spectre pollinique des miels : 60 formes y sont identifiées dont 50 de plantes mellifères et 10 de plantes non mellifères. Le nombre moyen des formes polliniques par miel (plantes non mellifères incluses) s'élève à 21. Les nombres de formes polliniques dans les miels se situent dans une large gamme de catégories, de 6-10 formes à 31-35 formes avec un maximum d'échantillons possédant de 16 à 25 formes (Fig. 2). Le Tableau II exprime la afréquence de distribution" des taxons dans les miels de l'Ontario. Parmi les taxons mellifères, la classe des pollens très fréquents (>50\%) comprend 12 taxons, celle des pollens fréquents (20-50\%) renferme 18 taxons; les pollens peu fréquents (10$20 \%$ ) comprennent 10 taxons et les pollens rares $(<10 \%), 11$ taxons. Parmi les taxons non mellifères (Tabl. III), 3 types polliniques sont fréquents, 3 formes polliniques sont peu fréquentes et 4 rares. Dans le Tableau IV, les formes polliniques abondantes dans les miels sont classées par ordre de fréquence décroissante. Toutes, sauf Tilia, appartiennent à la classe des pollens très fréquents (> 50\%). Trois taxons atteignent la classe de pollens dominants ( $>45 \%$ du pollen d'un même taxon dans un miel) : Lotus, Trifolium hybridum / T. repens et les Brassica-

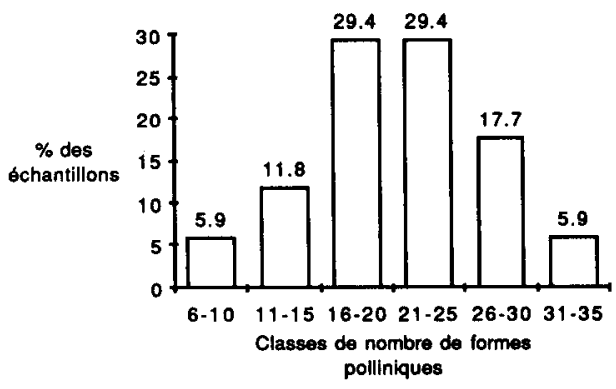

Fig. 2. Histogramme de fréquence du nombre de formes polliniques dans les miels de l'Ontario. ceae (autres). Le Tableau V donne le CAP-10 des miels unifloraux relevés. II est à noter que le miel de Tilia est normalement sous-représenté au point de vue pollinique (Louveaux et al., 1978). Les indicateurs de miellat sont réduits : $50 \%$ des miels en renferment peu (nombre d'indicateurs de miellat/nombre de pollens ou $\mathrm{IM} / \mathrm{P}=0,10-1,49)$ et $50 \%$ très peu (IM/P $<0,09$ ) (Tableau I).

\section{Discussion et conclusion}

Les principales sources de miel dans le sud de l'Ontario sont : Lotus et Trifolium hybridum / T. repens dont les dominances polliniques sont les plus nombreuses. $\mathrm{Ce}$ sont aussi les plus caractéristiques car leurs pollens sont présents dans tous les miels analysés. Les Brassicaceae (autres), dont un seul cas de dominance a été observé, y sont moins caractéristiques car aucun cas de pollen d'accompagnement n'a été relevé et la fréquence de ce taxon n'atteint que $64,7 \%$ des échantillons. L'abondance dans les miels des autres pollens très fréquents ( $>50 \%$ ) est variable : ceux de Melilotus et du type Solidago entrent dans la classe des pollens d'accompagnement, ceux de Salix, Trifolium pratense, arbres fruitiers, Rubus, Rhus typhina et Rosaceae (repliées) dans la classe des pollens isolés importants et ceux de Brassica kaber restent dans la classe des pollens isolés rares. Par ailleurs, une forme pollinique peu fréquente, Tilia, atteint la classe des pollens d'accompagnement.

Un peu moins de la moitié des miels sont unifloraux ( 8 sur 17 ou $47 \%$ ), les autres $(53 \%)$ étant des miels «toutes fleurs", c'est-à-dire sans dominance pollinique. Ceux-ci comportent les pollens d'accompagnement de un, deux ou trois des taxons suivants : Lotus, Trifolium 
Tableau I. Classes de fréquence des formes polliniques de plantes mellifères (en lettres capitales) et de plantes non mellifères (en minuscules) dans chaque miel provenant de l'Ontario, Canada.

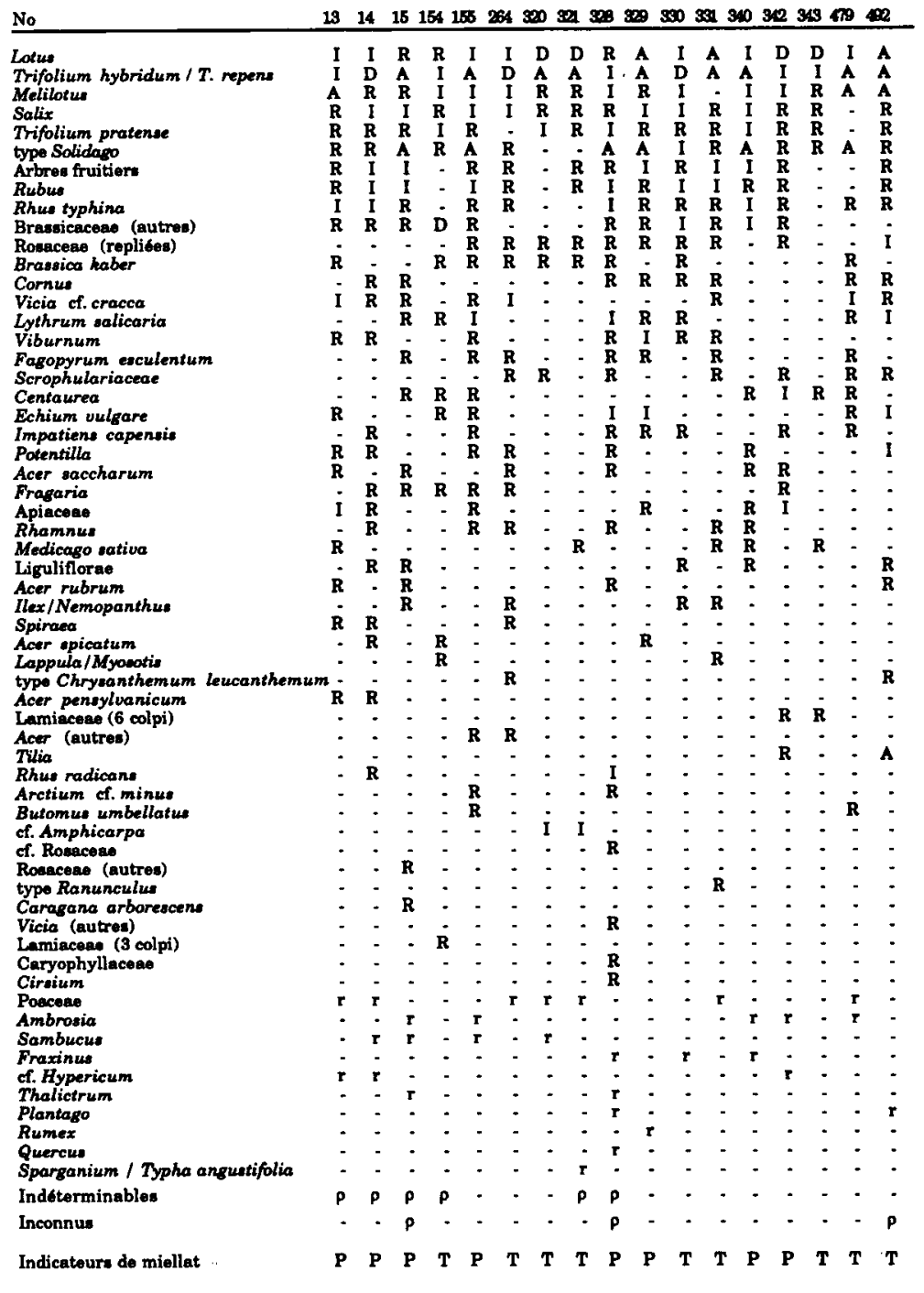

Les pollens non déterminés sont indiqués par des lettres grecques.

$\mathrm{D}=$ pollen dominant $(>45 \%) ; \mathrm{A}=$ pollen d'accompagnement $(16-45 \%) ; \mathrm{I}=$ pollen isolé important $(3-15 \%) ; \mathrm{R}=$ pollen isolé $(<3 \%)$.

Les classes de fréquence des indicateurs de miellat donnent le rapport du nombre dindicateurs de miellat (IM) au nombre total de grains de pollen de plantes productrices de nectar $(P) ; T=$ pratiquement aucun $(I M / P<0,10) ; P=$ peu (IMP $=0,10-1,49$ ).

Pour la localisation des sites, voir Fig. 1. 
Tableau II. Fréquence de distribution des pollens de plantes mellifères dans les miels de l'Ontario.

Pollens tres fréquents (> 50\%)
Lotus
Trifolium hybridum / T. repens
Melilotus
Salix
Trifolium pratense
type Solidago
Arbres fruitiers
Rubus
Rhus thyphina
Brassicaceae (autres)
Rosaceae (repliées)
Brassica kaber
Pollens frequents (20-50\%)
Cornus
Vicia cf. cracca
Lythrum salicaria
Viburnum
Fagopyrum esculentum
Scrophulariaceae
Centaurea
Echium vulgare
Impatiens capensis
Potentilla
Acer saccharum
Fragaria
Apiaceae
Rhamnus
Medicago sativa
Liguliflorae
Acer rubrum
lex / Nemopanthus
Pollens peu fréquents (10-20\%)
Spiraea
Acer spicatum
Lappula / Myosotis
type Chrysanthemum leucanthemum
Acer pensylvanicum
Lamiaceae (6 colpi)
Acer (autres)
Tilia
Rhus radicans
Arctium cf. minus
Butomus umbellatus
cf. Amphicarpa
Pollens rares (< 10\%)
cf. Rosaceae
Rosaceae (autres)
type Ranunculus
Caragana arborescens
Vicia (autres)
Lamiaceae (3 colpi)
Caryophyllaceae
Cirsium

lotier

tréfle alsike / t. blanc

mélilot

saule

trèfle rouge

type verge d'or

arbres fruitiers

ronce ou framboisier

vinaigrier

Crucifieres

Rosacées

moutarde des champs

comouiller

vesce cf. jargeau

salicaire

viorne

sarrasin

Scrophulariacées

centaurée

vipérine

impatiente du cap

potentille

érable à sucre

fraisier

Ombellifères

nerprun

luzerne

Liguliflores

érable rouge

houx / faux houx

spiréo

erable à épis

bardanette / myosotis

type marguerite

érable de Pennsylvanie

Labiées (6 colpi)

érable

tilleul

sumac grimpant

bardane of. mineure

butome à ombelles

cf. amphicarpe

cf. Rosacées

Rosacées

renoncule

acacia jaune

vesce

Labiées ( 3 colpi)

Caryophyllacées

chardon
100,00

100,00

94,12

94,12

88,24

88,24

76,47

76,47

76,47

64,71

58,82

52,94

47,06

47,06

47,06

41,18

41,18

41,18

41,18

41,18

41,18

41,18

35,29

35,29

35,29

35,29

29,41

29,41

23,53

23,53

17,65

17,65

11,76

11,76

11,76

11,76

11,76

11,76

11,76

11,76

11,76

11,76

5,88

5,88

5,88

5,88

5,88

5,88

5,88

5,88 
Tableau III. Fréquence de distribution des pollens de plantes non mellifères dans les miels de I'Ontario.

\begin{tabular}{|c|c|c|}
\hline Taxons & & $\%$ \\
\hline $\begin{array}{l}\text { Pollens fréquents }(\mathbf{2 0 - 5 0 \% )} \\
\text { Poaceae } \\
\text { Ambrosia } \\
\text { Sambucus }\end{array}$ & $\begin{array}{l}\text { Graminées } \\
\text { ambroisie } \\
\text { sureau }\end{array}$ & $\begin{array}{l}41,18 \\
29,41 \\
23,53\end{array}$ \\
\hline $\begin{array}{l}\text { Pollens peu fréquents }(10-20 \%) \\
\text { Fraxinus } \\
\text { cf. Hypericum } \\
\text { Thalictrum } \\
\text { Plantago }\end{array}$ & $\begin{array}{l}\text { frêne } \\
\text { cf. millepertuis } \\
\text { pigamon } \\
\text { plantain }\end{array}$ & $\begin{array}{l}17,65 \\
17,65 \\
11,76 \\
11,76\end{array}$ \\
\hline $\begin{array}{l}\text { Pollens rares }(<10 \%) \\
\text { Rumex } \\
\text { Quercus } \\
\text { Sparganium / Typha angustifolia }\end{array}$ & $\begin{array}{l}\text { rumex } \\
\text { chêne } \\
\text { rubanier/quenouille }\end{array}$ & $\begin{array}{l}5,88 \\
5,88 \\
5,88\end{array}$ \\
\hline
\end{tabular}

hybridum / T. repens, Melilotus et du type Solidago. Dans un cas Tilia s'ajoute à Lotus et à Trifolium hybridum / T. repens. Ce miel, où le pollen de Tilia atteint $22 \%$ des pollens présents, peut être considéré comme miel de tilleul car ces pollens sont normalement sous-représentés (Louveaux et al., 1978). Le CAP-10 de l'échantillon de 22500 grains entre dans les normes décrites par Maurizio (1958) et

Tableau IV. Types polliniques dominants (D) ou d'accompagnement (A) présents dans les miels de l'Ontario.

\begin{tabular}{|c|c|c|c|c|c|c|c|c|c|c|c|}
\hline \multirow{3}{*}{$\begin{array}{l}\text { Types } \\
\text { polliniques }\end{array}$} & \multicolumn{8}{|c|}{$\begin{array}{l}\text { Nombre d'échantillons où le type pollinique } \\
\qquad e s t(D) \text { ou }(A)\end{array}$} & \multirow[t]{3}{*}{$\begin{array}{c}\text { Nombre } \\
\text { d'échantillons } \\
\text { où la forme } \\
\text { est présente }\end{array}$} & \multicolumn{2}{|c|}{$\begin{array}{c}\% \text { des } \\
\text { échantillons } \\
\text { où la forme } \\
\text { est }(D) \text { ou }(A)\end{array}$} \\
\hline & \multicolumn{2}{|c|}{ ouest } & \multicolumn{2}{|c|}{ centre } & \multicolumn{2}{|c|}{ èst } & \multicolumn{2}{|c|}{ Total } & & & \\
\hline & $D$ & $A$ & $D$ & $A$ & $D$ & $A$ & $D$ & $A$ & & $D$ & $A$ \\
\hline Lotus & 4 & - & - & 3 & - & - & 4 & 3 & 17 & 23,5 & 17,6 \\
\hline $\begin{array}{l}\text { Trifolium hybridum / } \\
\text { T. repens }\end{array}$ & 1 & 3 & 1 & 4 & 1 & 2 & 3 & 9 & 17 & 17,6 & 52,9 \\
\hline Melilotus & - & - & - & - & - & 2 & - & 2 & 16 & - & 12,5 \\
\hline type Solidago & - & 1 & - & 3 & - & 2 & - & 6 & 15 & - & 40,0 \\
\hline Brassicaceae (autres) & - & - & - & - & 1 & - & 1 & - & 11 & 9,1 & - \\
\hline Tilia & - & - & - & 1 & - & - & - & - & 2 & - & 50,0 \\
\hline
\end{tabular}


par Demianovicz (1964) pour les miels de ce genre. La production de nectar par cette plante est irrégulière et occasionnelle mais très importante lorsqu'elle a lieu (Townsend et Burke, 1952; Jamieson, 1958; Pellet, 1977). Ceci permet d'expliquer la rareté de ce taxon présent dans 2 miels seulement, bien que Tilia americana soit assez fréquent en Ontario sur des terrains boisés et le long de clôtures (Townsend et Burke, 1952). Il est une des espèces importantes à bois dur des forêts de feuillus du sud de l'Ontario (Ramsay, 1987). Pellet (1977) considère le tilleul comme une source importante de nectar. Austin (1958) et Louveaux (1966) le considèrent comme caractéristique des miels de l'Ontario.

Les miels de Lotus de notre échantillonnage (Tableau $V$ ) renferment un CAP-10 de $18 \quad 611$ à 69160 grains, teneurs moyennes qui concordent avec celles relevées par Maurizio et Louveaux (1965) pour des miels de lotier d'Europe. L'analyse de 7 miels ontariens effectuée par Adams et al. (1979 et 1981) et provenant de la région de Guelph n'a mis en évidence pour ce taxon que des valeurs équivalant à des "pollens d'accompagne- ment». Notre étude met donc en évidence pour la première fois la présence de miel de Lotus en Ontario. Elle confirme aussi l'importance que Adams et al. (1979 et 1981) ont accordé à ce taxon comme source de nectar en juillet-août en Ontario. II s'agit sans aucun doute de Lotus corniculatus dont la culture et celle de Medicago sativa ont remplacé celles de Trifolium hybridum et de Melilotus depuis une cinquantaine d'années (Crane, 1966). On a longtemps mis en doute la valeur mellifère de Lotus corniculatus en Ontario (Adams et al., 1979), aussi cette plante n'a-t-elle été mentionnée qu'exceptionnellement parmi les plantes mellifères recommandées pour l'apiculture dans cette province (Jamieson, 1958; Ramsay, 1987).

Le CAP-10 des miels de Trifolium hybridum / T. repens de notre échantillonnage qui se classe dans les catégories à teneurs polliniques moyennes et riches varie de 34444 à 109166 grains. Ces valeurs sont donc proches et dépassent peu les données fournies par Maurizio et Louveaux (1965) de 27000 à 90000 grains $/ 10 \mathrm{~g}$ de miel. Trifolium hybridum / $T$. repens, considéré comme une des principales sources de nectar (Pellet, 1977;

Tableau V. Nombre de grains de pollen par $10 \mathrm{~g}$ de miel (CAP-10) dans les miels unifloraux de l'Ontario.

\begin{tabular}{|c|c|c|c|c|c|}
\hline \multirow[t]{2}{*}{$\begin{array}{l}\text { Pollen *dominant» } \\
\text { (PD) }\end{array}$} & \multirow[t]{2}{*}{$\begin{array}{c}\text { Amplitude } \\
d \theta s \% d \theta P D\end{array}$} & \multicolumn{3}{|c|}{$\begin{array}{l}\text { Nombre d'échantillons dans } \\
\text { chaque catégorie de CAP-10 }\end{array}$} & \multirow[t]{2}{*}{$\begin{array}{l}\text { Nombre total } \\
\text { d'échantillons }\end{array}$} \\
\hline & & $<20000$ & $\begin{array}{l}20000 \\
-100000\end{array}$ & $\begin{array}{l}100000 \\
-500000\end{array}$ & \\
\hline Lotus & $60,0-84,0$ & 1 & 3 & - & 4 \\
\hline $\begin{array}{l}\text { Trifolium hybridum / } \\
\text { T. repens }\end{array}$ & $51,3-56,7$ & - & 2 & 1 & 3 \\
\hline Brassicaceae (autres) & 71,3 & - & - & 1 & 1 \\
\hline Tilia & 22,0 & 1 & - & - & 1 \\
\hline
\end{tabular}


Jamieson, 1958; Townsend et Burke, 1952), figure comme miel unifloral dans les analyses de Louveaux (1966). Nos résultats confirment son rôle prédominant dans les miels de l'Ontario.

Les miels de Lotus corniculatus n'ont été relevés que dans l'ouest de la région et en paraissent caractéristiques, tandis que des miels de Trifolium hybridum / $T$. repens ont été identifiés sur tout le territoire (Tableau IV).

Le miel de Brassicaceae (autres) de cet échantillonnage est riche en pollen $($ CAP-10 = 151 944); ceci concorde avec les données de la littérature (Maurizio, 1958) et il s'agit certainement de miel de colza connu pour sa richesse en pollen. Ce cas isolé de miel de Brassicaceae (autres), relevé dans le centre de la région, peut être considéré comme fortuit car aucun cas de pollen d'accompagnement de ce taxon n'a été observé dans l'ensemble des échantillons. Aucune mention de ce taxon n'est faite pour l'Ontario par les auteurs. Melilotus est cité par Pellet (1977) comme source mineure de miel mais est considéré comme source de surplus par Townsend et Burke (1952), Jamieson (1958) et Austin (1958). La culture du mélilot n'ayant plus l'importance d'autrefois (Ramsay, 1987), il est normal que ce taxon n'a que peu contribué à la production des miels de notre échantillonnage. Si son pollen est présent dans presque tous les échantillons, il n'atteint une certaine abondance que dans 2 cas comme pollen d'accompagnement. Solidago et Aster représentés par le type Solidago sont recommandés par Pellet (1977) comme sources de miel de fin de saison pour cette province. Townsend et Burke (1952) les considèrent comme plantes mineures et Jamieson (1958) ne les cite pas. Leur pollen ne figure qu'exceptionnellement dans les analyses polliniques de Louveaux (1966). II est cependant très fréquent dans notre échantillonnage et atteint la classe de pollen d'accompagnement dans 5 cas. $\mathrm{Ce}$ taxon semble donc apporter une participation valable aux miels de l'Ontario.

Parmi les pollens isolés $(<16 \%$ du même taxon dans un miel), Echium vulgare est caractéristique des miels de l'est car son pollen est absent des autres miels de la région. Pellet (1977) le cite comme source mineure de miel. Townsend et Burke (1952) considèrent cependant Echium vulgare comme plante mellifère localement importante et Ramsay (1987) comme source importante de nectar dans la vallée de l'Outaouais, ce que confirme notre étude. Les pollens de Viburnum, de Lythrum salicaria et de Cornus, non rapportés dans la littérature, sont très fréquents (> $50 \%$ des échantillons) dans les miels du centre et de l'est, dans des territoires limitrophes du lac Ontario et du fleuve Saint-Laurent jusqu'à la Rivière des Outaouais. Pellet (1977) cite comme autre source importante de nectar, le sarrasin dont le pollen a été relevé dans 7 miels de notre échantillonnage mais en quantité très faible de pollen isolé rare. Selon Townsend et Burke (1952) et Jamieson (1958), cette plante est cultivée localement en Ontario et peut y être source de miel. Parmi les sources mineures de miel citées par Pellet (1977), l'eupatoire (Eupatorium) qui appartient au type Solidago pourrait figurer dans notre échantillonnage, le chardon des champs (Cirsium arvense) n'a été relevé que très rarement et l'asclépiade (Asclepias syriaca), qui est très fréquente dans cette région, ne peut être décelée directement par analyse pollinique parce que le pollen de cette plante reste groupé en pollinies. Le CAP-10 très bas de plusieurs miels révèle la possibilité de l'apport du nectar de cette plante à la production du miel en Ontario. Les pollens de quelques espèces printanières recommandées pour l'apiculture, tels les érables, le saule, le pissenlit 
et les arbres fruitiers (Pellet, 1976; Townsend et Burke, 1952) ont été observés dans notre relevé.

Le nombre moyen de formes polliniques par miel (21) est plus élevé dans le sud de l'Ontario que dans les miels des provinces voisines (15 au Québec - Feller-Demalsy, 1983; en moyenne 9 dans les provinces des Prairies - Feller-Demalsy et al., 1987, a et b). Une plus grande diversité de la végétation due à la situation plus méridionale de cette région en est probablement la cause. Des observations similaires ont déjà été faites par Lieux (1981) et nous-même pour des miels d'autres régions (Feller-Demalsy, 1979 et 1983; Feiler-Demalsy et al., 1987, a). Par ailleurs, le nombre de formes polliniques dans les miels paraît plus bas à l'ouest de cette région, là où la culture est pratiquée à grande échelle, qu'à l'est, où dominent les pâturages (18 par rapport à 23). Une réduction du nombre de types polliniques dans les miels provenant de régions à culture intensive a aussi été observée dans les miels du Québec (Feller-Demalsy et Lamontagne, 1979 et Feller-Demalsy, 1983) et dans les miels du sud de la Saskatchewan (Feller-Demalsy et al., 1987, b). Dix-sept pour cent des formes polliniques relevées dans les miels appartiennent à des taxons non mellifères. Ces pollens sont plus répandus dans les miels de l'Ontario où ces formes sont rencontrées dans $88 \%$ des échantillons contre moins de $45 \%$ dans les provinces des Prairies (Feller-Demalsy et al., 1987, a et b; Feller-Demalsy et al., sous presse). La moyenne du nombre de formes polliniques non mellifères par miel s'élève à 1,6 dans les miels de l'Ontario; elle est supérieure à celle des Prairies, surtout à celle de l'Alberta et de la Saskatchewan où elle est inférieure à 1 (FellerDemalsy et al., 1987 a et b). Les indicateurs de miellat sont peu nombreux dans les miels de l'Ontario comme dans ceux des Prairies (Feller-Demalsy et al., 1987 a et b; Feller-Demalsy et al., sous presse). Peu d'algues y ont été relevées.

Dans le sud de l'Ontario, les miels sont caractérisés par des types polliniques appartenant principalement aux Fabaceae, en particulier Lotus corniculatus. Bien que dépendants en grande partie de plantes de culture, ces miels trouvent également une partie de leur source dans des plantes vivaces non cultivées (Tableau VI). Par leur composition florale, ils se rapprochent à la fois des miels de l'ouest du Québec, surtout du sud de l'Outaouais, et de certains miels de l'est du

Tableau VI. Caractéristiques polliniques des miels de l'Ontario.

\begin{tabular}{lll}
\hline Caractéristiques générales & Lotus corniculatus *** & \\
& Trifolium hybridum $/$ T. repens *** & \\
& Melilotus * & \\
& Salix & \\
& Trifolium pratense & \\
& type Solidago * & \\
& Echium vulgare & \\
Caractéristiques locales & Viburnum & Est-East \\
& Cythrum salicaria & Région limitrophe du lac \\
& Cornus & Ontario et du fleuve St- \\
& Laurent \\
Caractéristiques occasionnelles americana & \\
& Brassicaceae ** & \\
\hline
\end{tabular}

\footnotetext{
"* cas de "pollens dominants"; " cas de "pollens d'accompagnement".
} 
Manitoba, où le lotier est présent, voire dominant (Feller-Demalsy, 1983; FellerDemalsy et al., 1988) mais en diffèrent par un nombre plus élevé de taxons dans une partie des échantillons.

\section{Remerciements}

Nous adressons nos plus vifs remerciements au Dr. Phil Burke, "Provincial Apiarist» et professeur à l'Université de Guelph, Ontario, qui nous a fourni une partie des échantillons de cette étude et au Dr. Maurice V. Smith, professeur à l'Université de Guelph, pour l'appui fourni à ce projet. Ce travail a été financé par une subvention du Conseil des recherches en pêche et agro-alimentaire du Québec.

\section{Références}

Adams J.R., Smith M.V. \& Townsend G.F. (1979) Identification of honey sources by pollen analysis of nectar from the hive. J. Apic. Res. 18 (4) 292-297

Adams J.R. \& Smith M.V. (1981) Seasonal pollen analysis of nectar from the hive and of extracted honey. J. Apic. Res. 20 (4), 243-248

Austin G.H. (1958) Maltose content of honeys and its probable effects on Crystallization. Proceedings Tenth International Congress of Entomology, Montréal 4, 1001-1006

Benton W. (publisher) (1968) Encyclopedia Britanica 16. Encyclopedia Britanica Inc.

Birks H.J.B. (1973) Past and present vegetation of the Isle of Skye. A palaeoecological study. Cambridge University Press, London

Comité Permanent Canadien des Noms Géographiques (1974) Répertoire géographique du Canada, Ontario. Ministère de l'énergie, des mines et des ressources, Division des levés et de la cartographie, Ottawa

Crane E. (1966) Canadian Beekeeping Journey. Bee World 47, 55-65, 132-148

Demianovicz Z. (1964) Charakteristik der Einartenhonige. Ann. Abeille 7 (4), 273-288

Feller-Demaisy M.J. \& Lamontagne Y. (1979) Analyse pollinique des miels du Québec. Apidologie 10 (4), 313-340
Feller-Demalsy M.J. (1983) Le spectre pollinique des miels du Québec. Apidologie 14 (3), 147-174

Feller-Demalsy M.J., Parent J. \& Strachan A.A. (1987a) Microscopic analysis of honeys from Alberta, Canada. J. Apic. Res. 26 (2), 123-132

Feller-Demalsy M.J., Parent J. \& Strachan A.A. (1987b) Microscopic analysis of honey from Saskatchewan, Canada. J. Apic. Res. 26 (4), 247-254

Feller-Demalsy M.J., Parent J. \& Strachan A.A. (1988) Microscopic analysis of honeys from Manitoba, Canada. J. Apic. Res. (sous presse)

Jamieson C.A. (1958) Facts about beekeeping in Canada. Bee World 39 (9), 232-236

Lieux M.H. (1981) An Analysis of Mississippi (USA) Honey : pollen, color and moisture. Apidologie 12 (2), 137-158

Louveaux J. (1966) Pollenanalyse einiger kanadischer Honige. Z. Bienenforsch. 8, 195-202

Louveaux J., Maurizio A. \& Vorwohl G. (1978) Methods of Melissopalynology. Bee World 59 (4), 139-157

Maurizio A. (1958) Beiträge zur quantitativen Pollenanalyse des Honigs. 3. Absoluter Gehalt Pflanzlicher Bestandteile in Esparsette, Luzerne, Orangen und Rapshonigen. Ann. Abeille $11,93-106$

Maurizio A. \& Louveaux J. (1965) Pollens de plantes mellifères d'Europe. Union des Groupements apicoles français, Paris

Moore P.D. \& Webb J.A. (1978) An Illustrated guide to Pollen Analysis. Halsted Press, New York

Pellet F.C. (1977) American Honey Plants. Dadant \& Sons, Hamilton, Illinois

Ramsay J. (1987) Plants for Beekeeping in Canada and the northern USA. international Bee Research Association, London

Simpson-Lewis W., Moore J.E., Pocock N.J., Tayior M.C. \& Swan H. (1980) Les terres de choix du Canada : une étude sélective de l'utilisation des terres dans une perspective nationale. Dossier cartographique $n^{\circ} 4$. Direction générale des terres. Environnement Canada, Ottawa

Statistique Canada (1984) Production de miel, 1983. Division de la statistique agricole. Unité de l'horticulture. Catalogue 23-007, février

Townsend G.F. \& Burke P.W. (1952) Beekeeping in Ontario. Ministery of Agriculture and Food. Publ. 490 